\title{
X-ray Crystallography to Cryo-Electron Microscopy: Computing Infrastructure in Structural Biology
}

Jason Key ${ }^{1}$, Peter A. Meyer ${ }^{1}$, Carol Herre ${ }^{1}$, Michael Timony ${ }^{1}$, Dimitry Filonov ${ }^{1}$, Justin O'Connor ${ }^{1}$, and Piotr Sliz ${ }^{1,2}$

${ }^{1}$ Harvard Medical School, Boston MA, ${ }^{2}$ Boston Children's Hospital, Boston MA

SBGrid is an academic research group based at Harvard Medical School dedicated to structural biology computing. Started in 2000, the group's primary initiatives are the SBGrid consortium, a software collaborative of more than 320 structural biology labs worldwide (Morin, 2013), and the SBGrid DataBank which was established in 2015 as an open research data publication system for the Structural Biology community (Meyer, 2016). The SBGrid consortium provides a supported collection of scientist-created software titles for Crystallography, NMR, Electron Microscopy, Computational Chemistry, and Structure Visualization \& Analysis with workshops and webinars to connect scientists with the scientist-developers who create the software. SBGrid DataBank supports validation and reproduction of macromolecular models and development of structural biology processing methods (Meyer, 2016). It currently contains primary data sets supporting over 400 macromolecular structures. Recent advances in cryo-electron microscopy (cryo-EM) detector technology have resulted in an increasing number of X-ray crystallographers turning to cryo-EM to determine structures of biological macromolecules too intractable or too difficult to determine by crystallographic techniques. The computational infrastructure used to support x-ray crystallography is only marginally capable supporting cryo-EM computations. In particular, efficient cryo-EM computation requires more, and higher performance storage; and places significant emphasis on GPU computation. Additionally, the general principles that making primary data available supports structural validation and methods development applies even more significantly to cryo-EM than it has with $\mathrm{x}$-ray crystallography. SBGrid has long been at the forefront of supporting applications for structure determination using electron microscopes (cf. Cheng, 2004; Gonen, 2004; Fotin, 2004). Here we will present how various parts of SBGrid infrastructure including software, data management tools, as well as local and cloud high performance computing resources contribute to establishing a robust support for crystallography and cryo-EM experiments.

\section{References}

Morin, A. et al.,. Cutting Edge: Collaboration gets the most out of software. eLife 2:e01456 (2013).

Meyer, P.A. et al, Data Publication with the Structural Biology Data Grid Supports Live Analysis. Nature Communications, 7: 10882 (2016), 1-12.

Cheng $\mathrm{Y}$, Zak O, Aisen P, Harrison SC, Walz T. Structure of the human transferrin receptor-transferrin complex. Cell. 2004 Feb 20;116(4):565-76.

Gonen T, Sliz P, Kistler J, Cheng Y, Walz T. Aquaporin-0 membrane junctions reveal the structure of a closed water pore. Nature. 2004 May 13;429(6988):193-7.

Fotin A, Cheng Y, Sliz P, Grigorieff N, Harrison SC, Kirchhausen T, Walz T. Molecular model for a complete clathrin lattice from electron cryomicroscopy. Nature. 2004 Dec 2;432(7017):573-9. 